\section{AB0927 SUPERB MICROVASCULAR IMAGING COMPARED WITH POWER DOPPLER ULTRASOUND IN ASSESSING SYNOVITIS OF THE KNEE IN JUVENILE IDIOPATHIC ARTHRITIS: A PRELIMINARY STUDY}

Deniz Alis ${ }^{1}$, Burak Caglar Erol ${ }^{2}$, Serkan Akbas ${ }^{2}$, Amra Adrovic ${ }^{3}$, Mehmet Yildiz ${ }^{3}$, Kenan Barut ${ }^{3}$, Ozgur Kasapcopur ${ }^{3}$, ibrahim Adaletli ${ }^{2}{ }^{1}$ Istanbul Mehmet Akif Ersoy Thoracic and Cardiovascular Surgery Training and Research Hospital, Radiology, Istanbul, Turkey, ${ }^{2}$ Istanbul University Cerrahpasa, Radiology, Istanbul, Turkey,

${ }^{3}$ Istanbul University Cerrahpasa, Pediatric Rheumatology, Istanbul, Turkey

Background: Juvenile idiopathic arthritis (JIA) is a complex entity and is the most common rheumatologic disease of the children with the knee being the most frequently affected joint. Extensive proliferation of the synovial cells, synovial thickening and inflammation are the hallmark pathological processes ongoing in the affected joints. The traditional methods in assessing the disease activity involve investigating tenderness, pain, and swelling of the relevant joint on psychical examination in addition to biochemical markers; however, the psychical examination may yield equivocal results in some patients. Sonographic modalities are being increasingly used as a complementary method in the assessment of the joints in JIA and Power Doppler ulltrasound (PDUS) is the current reference sonographic method in assessing the inflammation by measuring the vascularity in the synovium. SMI is a novel Doppler technique using advanced filtering algorithms with an ability to detect even subtle and slow blood flow signals.

Objectives: we aimed to investigate the efficiency of Superb Microvascular imaging (SMI) in assessing inflammation of synovium in the knee of patients with JIA compared with PDUS.

Methods: Both knees of the patients with a diagnosis of clinically active JIA were examined using gray-scale US. The knees with positive US and physical examination findings included in group A while the knees with positive US findings despite negative physical examination findings included in group B. The observers calculated vascularity index (VI) by manually drawing a region of interest $(\mathrm{ROI})$ onto the thickest part of the synovium using PDUS and SMI.

Results: A total of 41 knees with both clinical and sonographic positive findings constituted group A and 14 knees with sonographic positive findings and normal physical examination categorized as group $B$ in the final cohort. The median SMI-VI (observer $1=4.9 \%$, IQR 3.6; observer $2=$ $4.1 \%$, IQR 4.6) was exceeded the median PDUS-VI (observer $1=1.5 \%$, IQR 1.8; observer $2=1.5$, IQR 1.9) $(\mathrm{P}<0.0001)$. In group $B$, the median SMI-VI (observer $1=2.85 \%$, IQR 8 ; observer $2=3.1 \%$, IQR 6.3) were exceeded the median VI PDUS-VI (observer $1=0.5 \%$, IQR 2; observer $2=0.55$, IQR 2.3) $(P=0.002$ and $P=0.001$ for observer 1 and observer 2 , respectively). No significant differences were observed between groups concerning PDUS-VI and SMI-VI $(P>0.05)$. In all of the patients in group $\mathrm{A}$, SMI was able to identify the presence of vascularity for both observers while no blood flow was detected $(\mathrm{VI}=0 \%)$ using PDUS in 6 patients (14.6\%) for observer 1 and 7 patients (17.1\%) for observer 2. In all of the patients in group B, SMI was also able to identify the presence of vascularity for both observers while no blood flow was detected (VI=0\%) using PDUS in 5 patients (\%35.7) for each observers.

Conclusion: SMI-VI obtained from the hypertrophied synovium of both clinically active and inactive knee joints were substantially higher compared with PDUS-VI in JIA patients. Furthermore, SMI detected the presence of blood flow in patients in whom PDUS could not be able to identify any blood flow. The findings of the present work indicate that SMI seems to a promising tool and a valuable adjunct to conventional US in assessing the inflammation of the synovial tissue in JIA patients.

\section{REFERENCES}

[1] Giancane G, et al. Juvenile Idiopathic Arthritis: Diagnosis and Treatment. Rheumatol Ther 2016;3:187-207

[2] Strunk J, et al. A new approach to studying angiogenesis in rheumatoid arthritis by means of power Doppler ultrasonography and measurement of serum vascular endothelial growth factor. Rheumatology 2004;43:14801483

Disclosure of Interests: None declared DOI: 10.1136/annrheumdis-2019-eular.2250

\section{AB0928 \\ CORONARY ARTERY LESIONS AT PRESENTATION IN KAWASAKI DISEASE: A MARKER OF IVIG RESISTANCE?A RETROSPECTIVE STUDY FROM NORTH INDIA}

MANJARI AGARWAL, Sumidha Mittal, Anju Singh, Sujata Sawhney. Sir Ganga Ram Hospital, Pediatric Rheumatology, NEW DELHI, India

Background: Kawasaki disease has been well described from all around the world.This has been well described from our country. We recently changed our practice with regards to addition of corticosteroids and undertook this study.

Objectives: To study the clinical profile of children with Kawasaki disease presenting to our unit.

To study the response of therapy in children with Kawasaki disease To study the efficacy of addition of upfront steroids in children with coronary artery lesions ( $z$ score $>2.5$ )

Methods: All children with Kawasaki disease seen in the last 5 years were included and data collected on prefilled proforma. IVIG $2 \mathrm{gm} / \mathrm{kg}$ as a single dose over 18-24 hours was given to all children. Aspirin in dose of $30-80 \mathrm{mg} / \mathrm{kg} /$ day was given to all children along with IVIG. Response was defined as defervescence within 36 hours of completion of IVIG. Fever beyond 36 hours of completion of IVIG was considered as no response/resistance to 1st dose of IVIG.

Results: 60 children diagnosed with Kawasaki disease were included. Median age at diagnosis of disease was 3.12 years(0.3-15 years).33 children had complete Kawasaki disease, 26 children had incomplete disease and one child had atypical Kawasaki disease36 children were diagnosed within first 10 days of disease onset and 24 children were diagnosed after day 10 of illness.18 children had history of infections prior to onset of Kawasaki disease.29 children(48\%) had coronary artery lesions at the time of presentation.48 children(80\%) were given 1 st dose of IVIG without any corticosteroids.36 children (75\%)responded to first dose of IVIG, one child was not given IVIG as he was diagnosed retrospectively and had self limiting disease. 14 children(29\%) did not respond to 1 st dose of IVIG. 9 children were given intravenous methylprednisolone $2 \mathrm{mg} / \mathrm{kg} /$ day in four divided doses along with IVIG. All of these responded to the first dose of IVIG. The size of coronary artery lesion also plays an important role. In children with $z$ score $>2.5$ the response to 1 st dose of IVIG was $61.5 \%$ whereas it was $94 \%$ in those who had $z$ scores $<2.5$ ( $p$ value 0.03 )

Abstract AB0928 Table 1. Response of therapy in children with and without coronary artery lesions

\begin{tabular}{lll}
\hline Group & No. & $\%$ \\
\hline No coronary artery lesion & $27 /$ & 90 \\
& 30 & 55 \\
Coronary artery lesion present, no steroids used & $11 /$ & 100 \\
Coronary artery lesion present, steroid given & 20 & \\
upfront & $9 / 9$ &
\end{tabular}

Response rate was significantly higher in patients with no coronary artery lesions ( $p$ value0.007).In children with coronary artery lesions at presentation, the response rate was significantly higher in children who were given steroids upfront ( $p$ value 0.027)

Conclusion: We see many children with coronary artery lesions at pre sentation. This seems to be an indicator of resistance to IVIG. It is beneficial to add steroids upfront along with IVIG to children with coronary artery lesions more than $2.5 \mathrm{z}$ score.

\section{REFERENCE}

[1] Dallaire F, Dahdah N. New equations and a critical appraisal of coronary artery Z scores in healthy children. J Am Soc Echocardiogr. 2011 Jan;24 (1):60-74.

Disclosure of Interests: None declared DOI: 10.1136/annrheumdis-2019-eular.4459 